\section{SAT0479 UPDATE FROM THE JUVENILE SCLERODERMA INCEPTION COHORT. WWW.JUVENILE-SCLERODERMA. COM}

Ivan Foeldvari ${ }^{1}$, Jens Klotsche ${ }^{2}$, Ozgur Kasapcopur ${ }^{3}$, Amra Adrovic ${ }^{3}$, Kathryn Torok ${ }^{3}$, Valda Stanevicha ${ }^{3}$, Flávio R. Sztajnbok ${ }^{3}$, Maria T. Tererri ${ }^{3}$, Ekaterina Alexeeva ${ }^{3}$, Jordi Anton ${ }^{3}$, Maria Katsikas ${ }^{3}$, Vanessa Smith ${ }^{3}$, Tadej Avcin ${ }^{3}$, Rolando Cimaz ${ }^{3}$, Mikhail Kostik ${ }^{3}$, Thomas Lehman ${ }^{3}$, Walter Alberto SifuentesGiraldo ${ }^{3}$, Simone Appenzeller ${ }^{3}$, Mahesh Janarthanan ${ }^{3}$, Monika Moll ${ }^{3}$, Dana Nemcova ${ }^{3}$, Maria Jose Santos ${ }^{3}$, Dieneke Schonenberg ${ }^{3}$, Cristina Battagliotti ${ }^{3}$, Lillemor Berntson ${ }^{3}$, Blanca Bica ${ }^{3}$, Juergen Brunner ${ }^{3}$, Patricia Costa Reis ${ }^{3}$, Despina Eleftheriou ${ }^{3}$, Liora Harel ${ }^{3}$, Gerd Horneff ${ }^{3}$, Tilmann Kallinich ${ }^{3}$, Dragana Lazarevic ${ }^{3}$, Kirsten Minden ${ }^{2}$, Susan Nielsen ${ }^{3}$, Farzana Nuruzzaman ${ }^{3}$, Anjali Patwardhan ${ }^{3}$, Yosef Uziel ${ }^{3}$, Nicola Helmus ${ }^{1} .{ }^{1}$ Hamburger Zentrum für Kinderund Jugendrheumatologie, Hamburg, Germany, ${ }^{2}$ Deutsches RheumaForschungszentrum (DRFZ), Berlin, Germany; ${ }^{3}$ jSSc Collaborative Group, Hamburg, Germany

Background: Juvenile systemic scleroderma (jSSc) is an orphan disease with a prevalence of 3 in 1000000 children. There are limited data regarding the clinical presentation of jSSc. The Juvenile Systemic Scleroderma Inception Cohort (JSSIC) is a multinational registry that prospectively collects information regarding patients with this disease in a standardized manner.

Objectives: Evaluation of the jSSc patients at the time of inclusion in the JSSIC

Methods: Patients were included in the JSSIC if they fulfilled the adult classification criteria, if they presented the first non Raynaud symptom before 16 years old and if they were younger than 18 years of age at the time of inclusion. Patients' characteristics at time of inclusion were evaluated.

Results: Currently, the cohort includes 120 patients, being 89\% Caucasian and $80 \%$ female. The majority had diffuse subtype (74\%) and $18 \%$ had overlap features. The mean age of onset of Raynaud phenomenon was 9.7 years in the diffuse subtype (djSSc) and

10.7 years in the limited subtype (liSSc). The mean age of non-Raynaud's symptoms was 10.0 years in the djSSc and 11.4 years in the ljSSc $(p=0.041)$. Mean disease duration at time of inclusion was 3.4 years in the djSSc and 2.4 years in the ljSSc group.

Mean Modified Rodnan skin score was 17.5 in the djSSc and 7.3 in the ljSSc $(p=0.002)$. Gottron papulae were significantly more common in the djSSc compared to ljSSc group ( $29 \%$ vs $6 \%$, respectively) ( $p=0.011)$. History of ulceration was significantly more common in the djSSc than in the ljSSc group (57\% vs $30 \%$, respectively) ( $p=0.004)$. $\quad F V C<80 \%$ occurred in $31 \%$ in the djSSc and $24 \%$ in the ljSSc group ( $p=0.55$ ). Pulmonary hypertension assessed by echocardiogram occurred around $7 \%$ in both groups. No systemic hypertension or renal crisis was reported. Gastrointestinal involvement occurred in $39 \%$ in the djSSc and in $26 \%$ in the ljSSc $(p=0.176)$. Number of joints with decreased range of motion was observed in approximately half of patients in both groups. Muscle weakness with joint contractures was present in $18 \%$ in the djSSc and $38 \%$ in the ljSSc group $(p=0.271)$. Tendon friction rub was present in $11 \%$ in djSSc and $4 \%$ in the ljSSc group. djSSc patients had significantly worse scores for physician global disease activity (VAS 0-100) (41vs 30) $(p=0.020)$ and for physician global disease damage (VAS $0-100)$ (37 vs 18) $(p=0.001)$. Patient judgment of disease activity and damage was similar in both subtypes. ANA positivity was $88 \%$ in both groups. Anti-Scl70 was positive in $33 \%$ in djSSc and $37 \%$ in the ljSSc group. Anticentromere positivity occurred in $3 \%$ in the djSSc and $10 \%$ in the ljSSc group. ESR was elevated in $30 \%$ in djSSc compared to $18 \%$ in the ljSSc group. DMARDs were used in $86 \%$ of the patients.

Conclusion: In this large cohort of jSSc patients there were surprisingly not many significant differences between djSSc and ljSSc. According to the physician global scores the djSSc patients have a significantly more severe disease.

Disclosure of Interests: Ivan Foeldvari Consultant for: Chugai, Novartis, Jens Klotsche: None declared, Ozgur Kasapcopur: None declared, Amra Adrovic: None declared, Kathryn Torok: None declared, Valda Stanevicha: None declared, Flávio R. Sztajnbok: None declared, Maria T. Tererri: None declared, Ekaterina Alexeeva: None declared, Jordi Anton Grant/ research support from: Grant/research support, consultant or speakers bureau from AbbVie, Alexion, Bristol-Myers Squibb, ChemoCentryx, Gebro, GlaxoSmithKline, Novartis, Novimmune, Pfizer, Roche, Sanofi and Sobi, Consultant for: Grant/research support, consultant or speakers bureau from AbbVie, Alexion, Bristol-Myers Squibb, ChemoCentryx, Gebro, GlaxoSmithKline, Novartis, Novimmune, Pfizer, Roche, Sanofi and Sobi, Speakers bureau: Grant/research support, consultant or speakers bureau from AbbVie, Alexion, Bristol-Myers Squibb, ChemoCentryx, Gebro,
GlaxoSmithKline, Novartis, Novimmune, Pfizer, Roche, Sanofi and Sobi, Maria Katsikas: None declared, Vanessa Smith: None declared, Tadej Avcin: None declared, Rolando Cimaz: None declared, Mikhail Kostik: None declared, Thomas Lehman: None declared, Walter Alberto Sifuentes-Giraldo: None declared, Simone Appenzeller: None declared, Mahesh Janarthanan: None declared, Monika Moll: None declared, Dana Nemcova: None declared, Maria Jose Santos: None declared, Dieneke Schonenberg: None declared, Cristina Battagliotti: None declared, Lillemor Berntson: None declared, Blanca Bica: None declared, Juergen Brunner: None declared, Patricia Costa Reis: None declared, Despina Eleftheriou: None declared, Liora Harel: None declared, Gerd Horneff: None declared, Tilmann Kallinich Grant/research support from: Novartis, Speakers bureau: Sobi, Roche, Novartis, CLB, Dragana Lazarevic: None declared, Kirsten Minden Consultant for: AbbVie, Susan Nielsen: None declared, Farzana Nuruzzaman: None declared, Anjali Patwardhan: None declared, Yosef Uziel: None declared, Nicola Helmus: None declared DOI: 10.1136/annrheumdis-2019-eular.2550

\section{SAT0480 CHRONIC KIDNEY DISEASE IN PATIENTS WITH JUVENILE SYSTEMIC LUPUS ERYTHYMATOSUS: EVIDENCE FROM A SINGLE CENTRE}

Yasmin Mahfouz ${ }^{1,2}$, Anastasia- Vasiliki Madenidou', Coziana Ciurtin ${ }^{1} .{ }^{1} U C L$, Arthritis Research UK Centre for Adolescent Rheumatology, London, United Kingdom; ${ }^{2}$ UCL, Medical School, London, United Kingdom

Background: Estimated 10- 20\% of all patients with systemic lupus erythematosus (SLE) develop clinical disease before the age of 18 years and are therefore classified as juvenile-onset SLE (JSLE). JSLE is characterised by a higher prevalence of lupus nephritis, compared to adult- onset SLE (1). Chronic kidney disease (CKD) refers to a state of irreversible kidney damage and/or reduction of kidney function that is associated with progressive loss of function over time. Lupus nephritis does not always lead to CKD. However, when it does it is associated with increased morbidity and mortality (2).

Objectives: We aimed to identify clinical and laboratory predictors of CKD development in JSLE patients by comparing the baseline characteristics of JSLE patients with and without CKD to ascertain if there are any significant differences between the two groups.

Methods: This is a single-centre retrospective study, who included patients reviewed in our young adult and adolescent clinics. All data were analyzed descriptively. Mann- Whitney $U$ or Chi- square test were performed to compare the characteristics between the patients with and without CKD. We used the Pearson's ( $r$ ) or Kendall's T (tau) correlation to examine if there is any association between the CKD and the baseline characteristics.

Results: We identified 44 JSLE patients, out of which 17 (39\%) fulfilled the diagnostic criteria for CKD at their last clinical review. The stages of CKD varied from 2 to 5 . All patients with CKD also had lupus nephritis, while $5 / 44$ patients $(11 \%)$ had lupus nephritis without CKD. The baseline characteristics are detailed in the table below. There were statistical significant differences in the treatments used for patients with and without CKD. As expected, the highest dsDNA levels were higher in patients with CKD $(p=0.03)$. There was also a positive moderate correlation $(\rho=$ 0.32 ) between raised levels of dsDNA and the development of CKD ( $p=$ $0.008)$. We also found a negative moderate correlation ( $T=-0.439)$ between the presence of RF and CKD ( $p=0.04)$.

Conclusion: Acknowledging the limitations posed by this small study, we identified a negative moderate correlation between the presence of RF and CKD, which has also been reported in the literature before (3). We cannot conclude that RF exerts a protective effect against renal disease in SLE, because of the many confounders that might account for a decreased RF in JSLE. Further research using a large JSLE cohort enabling multivariate logistic regression is recommended. DsDNA antibody levels are a measure of disease activity in lupus nephritis and therefore this might explain why patients who developed CKD were noted to have higher anti-dsDNA levels, in comparison with the patients who did not develop CKD.

\section{REFERENCES}

[1] Ambrose $\mathrm{N}$, et al. Differences in disease phenotype and severity in SLE across age groups. Lupus. 2016;25(14):1542-50.

[2] Hagelberg S, et al. Longterm followup of childhood lupus nephritis. J Rheumatol. 2002;29(12):2635-42.

[3] Helin $\mathrm{H}$, et al. Rheumatoid factor in rheumatoid arthritis associated renal disease and in lupus nephritis. Ann Rheum Dis. 1986;45(6):508-11 